\title{
Umfrage
}

\section{Dentale \\ Lokalanästhesie ist \\ für Zahnärzte und Patienten wichtig}

Die dentale Lokalanästhesie genießt in Deutschland bei Zahnärzten und Patienten gleichermaßen einen hohen Stellenwert. Das zeigen 2 Befragungen im Auftrag von Sanofi unter 1000 Männern und Frauen in Deutschland ab 14 Jahren sowie unter 100 Zahnärzten in Deutschland. Während für die Patienten bei der dentalen Lokalanästhesie die zuverlässige Schmerzreduktion im Vordergrund steht, profitieren die Zahnärzte von gelasseneren Patienten während der Behandlung. Neben dem positiven Effekt auf das Patientenwohlbefinden haben Zahnärzte weitere Anforderungen an ein Lokalanästhetikum: Wie die Befragung zeigt, sind vor allem Sicherheit und Wirkung wichtige Aspekte. Nicht zuletzt deshalb vertrauen Zahnärzte auf Ultracain $^{\circledR}$. Werden die Zahnärzte nach den ihnen bekannten und von ihnen verwendeten Lokalanästhetika befragt, nimmt Ultracain die Spitzenposition ein: Es ist das bekannteste und am häufigsten verwendete Lokalanästhetikum. 77 \% der Befragten verwenden es, $98 \%$ geben an, es zu kennen und aktuell zu verwenden beziehungsweise es schon verwendet zu haben.

Nach einer Pressemitteilung der Sanofi-Aventis GmbH, Frankfurt 